\title{
FUNDAMENTOS DE LAS SENTENCIAS CASATORIAS SOBRE TERCERÍA DE PROPIEDAD Y SUS IMPLICANCIAS FRENTE A LAS PRETENSIONES DE LA PARTE DEMANDANTE.
}

\author{
FUNDAMENTALS OF CASTORY JUDGMENTS ON THIRD PARTY PROPERTY AND ITS \\ IMPLICATIONS AGAINST THE PRETENSIONS OF THE CLAIMING PARTY
}

Lincoln Salas Ponce ${ }^{1}$

Carlos Alberto Pajuelo Beltrán²

Aceptado: $17 / 09 / 2019$

Publicado online:15/01/2020

\section{RESUMEN}

El problema radica en que muchas veces el tercerista no obtiene una sentencia favorable a sus pretensiones, e incluso en sede casatoria pocas veces se le reconoce su derecho. A pesar que el tercerista acredita con documento público o privado la propiedad del bien, otras circunstancias jurídicas procesales impiden que su derecho sea reconocido judicialmente. Precisamente, son las sentencias casatorias quienes nos proporcionan los fundamentos principales por el cual, el tercerista muchas veces no logra su pretensión. Se presenta el caso, de las sentencias casatorias correspondientes a procesos de tercerías de los años 2015-2016 nos han permitido conocer a cabalidad los fundamentos que inciden directamente en el fallo de la sentencia; asimismo, se puede conocer los aspectos principales que se dan en el debate judicial por la prevalencia del derecho real (propiedad) sobre el derecho personal (crédito) a pesar de que este último pueda estar debidamente inscrito. Investigación descriptiva, correlacional y explicativo. La investigación se ha desarrollado en una población de abogados civilistas de la jurisdicción de Tacna. Asimismo, se ha tenido en cuenta el análisis documental representado por las sentencias casatorias. Los instrumentos de medición han sido los cuestionarios y la guía de análisis documental. Se concluye que los principales

\footnotetext{
${ }^{1}$ Magíster en Derecho con Mención en Derecho Civil y Comercial, docente de la Facultad de Derecho y Ciencias Políticas de la Universidad Privada de Tacna, Tacna, Perú

2 Magíster en Derecho. Abogado especializado en materia aduanera. agente aduanas SUNAT, Tacna, Perú
} 
fundamentos de las sentencias casatorias analizados sobre tercería de propiedad están referidos a la inscripción registral, la prevalencia del derecho real de la propiedad sobre el derecho personal y requisitos de procedibilidad del recurso de casación, denotando un favorecimiento regular a los demandantes.

Palabras claves: Tercería, casación, propiedad, derecho real.

\section{ABSTRACT}

The problem lies in the fact that many times the third party does not obtain a sentence favorable to his claims, and even in cassary headquarters his right is rarely recognized. Although the third party accredits ownership of the property with a public or private document, other procedural legal circumstances prevent its right to be legally recognized. Precisely, it is the random judgments that provide us with the main foundations by which, the third party often fails to achieve his claim. In the present case, the random judgments corresponding to third-party proceedings of the years 2015-2016 have allowed us to fully understand the fundamentals that directly affect the judgment ruling; Likewise, it is possible to know the main aspects that occur in the judicial debate due to the prevalence of real law (property) over personal law (credit) even though the latter may be duly registered. Descriptive, correlational and explanatory research. The investigation has been carried out in a population of civil lawyers of the Tacna jurisdiction. Likewise, the documentary analysis represented by the judgments has been taken into account. The measuring instruments have been the questionnaires and the document analysis guide. It is concluded that the main grounds of the judgments analyzed on property third parties are related to the registration, the prevalence of the real property right over personal law and requirements for the appeal of cassation, denoting a regular favor to the plaintiffs .

Keywords: Third party, cassation, property, real right.

\section{INTRODUCCIÓN}

Las tercerías sean las de dominio o propiedad como las de mejor derecho, son instrumentos de protección que se encuentran a disposición de terceros afectados por un proceso de ejecución en el que no son parte, permitiéndoles hacer valer sus derechos e intereses y evitar de esta manera los efectos negativos de la ejecución que hasta la fecha las tercerías de propiedad siguen siendo poco eficaces en perjuicio de los terceros. Muchas veces la norma puede ser perfecta e imperfecta, sin embargo, es la conducta de la persona quien determina el resultado del proceso. 
La tercería en general, es la acción que compete a quien no es parte en el proceso, para atender sus derechos afectados con una medida cautelar o para evitar la ejecución o el derecho preferente de pago. Si el bien afectado jurídicamente por el juez con medida cautelar o para su ejecución no pertenece al deudor, sino a una persona ajena a la relación jurídica sustantiva existente entre el demandante-acreedor-y el demandado -deudor-; corresponde a ésta persona hacer valer su derecho de propiedad a efecto de que el juez, que dictó el gravamen, detenga la subasta pública, deje sin efecto tal medida y le entregue el bien libre del mencionado gravamen.

A pesar de que el tercerista acredita con documento público o privado la propiedad del bien, otras circunstancias jurídicas procesales impiden que su derecho sea reconocido judicialmente. Precisamente, son las sentencias quienes nos proporcionan los fundamentos principales por el cual, el tercerista muchas veces no logra su pretensión. Esta problemática no solamente afecta directamente a los terceristas por no lograr sus objetivos procesales con sentencias favorables, sino también a los jueces y abogados. Los jueces muchas veces tienen que tratar de adaptar la realidad de los hechos a las normas legales, entre ellos, la Constitución Política, el Código Civil, Código Procesal Civil, Jurisprudencias y Plenos Casatorios para finalmente emitir la sentencia respectiva, de los cuales reiteradamente dan interpretaciones contradictorias. Por su parte los abogados tratan de buscar la norma que más favorezca a los intereses de su patrocinado y se encuentran muchas veces -al igual que los jueces- con leyes implícitas y jurisprudencias contradictorias. Frente a esta problemática de las tercerías de propiedad, el legislador trata de regular y modificar las normas para que su interpretación sea clara, prueba de ello es la modificación que ha sufrido las normas respecto a las tercerías plasmados en el Código Procesal Civil. Igualmente, mediante los Plenos Casatorios se buscan unificar criterios para que la aplicación de la norma sea precisa. Sin embargo, los resultados para los terceristas son poco alentadores por no lograr que sus pretensiones sean favorables en sede casatoria. Las diversas posturas sobre la prevalencia de derechos han dado lugar a este problema, señalando muchas veces que el derecho real (propiedad) prevalece sobre el derecho personal (deuda) y teniendo como elemento vital la inscripción registral de esos derechos.

Lino Rodriguez en el 2013, concluye que "La ausencia de eficacia constitutiva auspicia la falta de utilidad social del registro, que queda indeterminado respecto de terceros en el momento en que se producen los cambios de propiedad y mantiene irresoluble el problema de la base física de los asientos registrales originando una realidad registral y otra extraregistral." Torres Mejía afirma que "Entre los principios procesales del derecho se encuentra el de igualdad, y es este principio el que le impone a los jueces la obligación de garantizar el derecho de defensa y mantener a las partes en los derechos y facultades comunes, esto incluye a los Terceros que si bien no son parte inicial del proceso pueden ser llamados a este a los fines de hacer valer sus derechos." (Décimo párrafo). Valdivieso Espinosa refiere que "La sustanciación del juicio ejecutivo en la práctica procesal civil ecuatoriana, permite evidenciar que existe la posibilidad de que se haga un uso doloso de la tercería coadyuvante, con la finalidad de favorecer al deudor moroso ejecutado y en detrimento de los intereses y derechos del acreedor ejecutante". 
En el Perú, los antecedentes del Recurso de Casación lo encontramos en el Código de Enjuiciamientos Civiles de 1852 donde se reguló sobre la base del "Recurso de Nulidad" español (Real Decreto de 04 noviembre de 1838), al haberse traducido literalmente el término francés "Casser" que podía ser interpretado como romper, destruir, anular; además, por su acepción española de "anular", y el Recours de Cassation (Recurso de Anulación o Recurso de Nulidad), como finalmente lo denominó la Ley de Enjuiciamiento Civiles de España del Siglo pasado.

Más adelante, se promulga el Código de Procedimientos Civiles (noviembre de 1911), que tuvo vigencia desde el 28 de julio de 1912 hasta la entrada del actual Código Procesal Civil (1993), se continuó bajo la misma denominación de Recurso de Nulidad, el cual es, una simple traducción literal del francés del Recurso de Casación, tal como sucedió con la legislación española de 1938, siendo en esencia en el origen la misma institución procesal que hoy tenemos legislada en los Arts. 384 y siguientes del Código Procesal Civil vigente. Rioja (2009) señala que la principal finalidad de este Recurso Extraordinario consiste en la defensa final de la voluntad de la ley, corregir los errores del juzgamiento y los errores en el procedimiento (error in iudicando y error in procedendo).

Aguila (2010) expresa que la casación "es un medio impugnatorio, que tiene un efecto revocatorio, pero también rescisorio, dependiendo de la causal que lo motiva. Se puede afirmar por ello, señala Monroy, que tiene naturaleza mixta." Ledesma (2008) señala que la casación "es un recurso en el que prima el interés público porque busca garantizar la vigencia de la ley y ser un órgano contralor del juzgador en los casos de violación de la norma jurídica o la jurisprudencia vinculante." Al respecto Celis (2013) refiere que el recurso de casación es esencialmente -conforme lo estatuía el Art. 384 del C.P.C.- un medio impugnatorio destinado a corregir errores in iudicando (respecto a la norma sustantiva objeto de la decisión) y errores in procedendo (respecto al procedimiento con el que adopta la decisión).

El término tercería proviene del latín: terciar que significa tercero. Ledesma (2008) refiere que la tercería "es un mecanismo de oposición a la ejecución por parte de tercero, ya sea porque este tercero acredita tener el derecho de propiedad de los bienes que han sido afectados por medida cautelar o para la ejecución; o porque es titular de un derecho de crédito preferente al del acreedor" y para lograr estos fines, se tramita como proceso abreviado.

Tal como señala Rioja (2011), las tercerías se caracterizan por ser un instrumento de protección previstas en nuestro ordenamiento jurídico y que se encuentran a disposición de terceros afectados por un proceso de ejecución en el que no son parte, permitiéndoles hacer valer sus derechos e intereses y evitar de esta manera los efectos negativos de la ejecución. Pasco (2014) confirma esta interpretación de la norma al señalar que procede la tercería frente a la ejecución de hipotecas sólo cuando el derecho de propiedad del tercerista hubiese sido inscrito con anterioridad a la constitución de la garantía. Hinostroza (2000) señala que el fundamento de la tercería excluyente de dominio radica en la concepción misma del derecho de propiedad: derecho real por el cual un bien se encuentra sometido a la voluntad y acción de una persona en forma 
exclusiva, pudiendo el dueño impedir a los demás su uso, goce o disposición. Siendo así, el proceso de tercería se basa en la protección de la propiedad (derecho real).

\section{OBJETIVOS}

a) Determinar cuál es el porcentaje de sentencias casatorias que en sus fundamentos contiene pronunciamiento de fondo sobre la fecha de inscripción registral de la propiedad y que han sido determinantes para el fallo de la sentencia.

b) Especificar cuál es el porcentaje de sentencias casatorias que en sus fundamentos contiene pronunciamiento de fondo sobre la prevalencia del derecho real sobre el derecho personal y que han sido determinantes para el fallo de la sentencia.

c) Determinar en qué medida las sentencias casatorias sobre procesos de tercería de propiedad favorecen a los demandantes.

\section{METODOLOGÍA}

Estudio descriptivo, correlacional, explicativo no experimental. La investigación se realizó en el ámbito de Tacna y abarcó el periodo 2015-2016. La muestra fue de 50 profesionales del Derecho. Para el análisis de las sentencias casatorias se utilizó la técnica del análisis documental. Para el trabajo de campo se utilizaron cuestionarios estructurados.

\section{RESULTADOS}

El 36\% de la muestra señala que las sentencias casatorias sobre proceso de tercería de propiedad favorecen regularmente a los demandantes, mientras que el $30 \%$ señalan que las sentencias casatorias mencionadas favorecen poco a los demandantes. El $42 \%$ señalaron que las sentencias casatorias sobre procesos de tercería de propiedad con pronunciamiento de fondo, tienen como principal fundamento la fecha de inscripción registral de la propiedad. El $44 \%$ consideraron que las sentencias tienen como principal fundamento la prevalencia del derecho real (propiedad) sobre el personal (crédito). El 90\% consideran que ante un conflicto entre el derecho real (propiedad) y derecho personal (crédito), debe prevalecer el derecho real. El 74\% considera que una inscripción de una medida cautelar de embargo prevalece sobre un derecho de propiedad no inscrito, pero con documento de fecha cierta anterior a la inscripción cautelar. El 94\% consideraron que si la inscripción registral de la transferencia de la propiedad inmueble fuese obligatoria (sistema constitutivo) en nuestro país, los procesos de tercería de propiedad (conflictos sobre la propiedad y los derechos personales) disminuirían considerablemente.

Respecto al análisis documental: Sentencias casatorias sobre tercerías de propiedad, se encontró que en el periodo 2015 - 2016, de las 13 sentencias casatorias sobre tercería de propiedad, 6 sentencias tuvieron como análisis de fondo lo referente a la fecha de inscripción registral (Tabla 01). 4 sentencias tuvieron como análisis de fondo la 
prevalencia del derecho real sobre el derecho personal (Tabla 02) 3 sentencias tuvieron como análisis de fondo otras causas que motivaron el fallo final, entre ellos el abandono del proceso, sobre carta de fianza y sobre el mandato de emisión de nueva resolución. (Tabla 3)

Tabla 1

Sentencias casatorias sobre prevalencia de fecha de inscripción registral de la propiedad

\begin{tabular}{|l|l|l|l|}
\hline $\mathrm{N}^{\circ}$ & $\mathrm{N}^{\circ}$ Expediente & Fallo & Favorecido \\
\hline 01 & $2826-2015$ AREQUIPA & INFUNDADO & Demandado \\
\hline 02 & $4592-2015$ LIMA SUR & INFUNDADO & Demandado \\
\hline 03 & $1144-2015$ CUSCO & FUNDADO & Demandado \\
\hline 04 & $925-2015$ LIMA ESTE & INFUNDADO & Demandado \\
\hline 05 & $2981-2015$ JUNIN & INFUNDADO & Demandado \\
\hline 06 & $3899-2015$ LIMA & INFUNDADO & Demandante \\
\hline
\end{tabular}

Tabla 2

Sentencias casatorias sobre prevalencia de derecho real sobre derecho personal

\begin{tabular}{|l|l|l|l|}
\hline $\mathrm{N}^{\circ}$ & $\mathrm{N}^{\circ}$ Expediente & Fallo & Favorecido \\
\hline 01 & $1821-2015$ LIMA & INFUNDADO & Demandante \\
\hline 02 & $112-2015$ LIMA & INFUNDADO & Demandante \\
\hline 03 & $26-2015$ LA LIBERTAD & INFUNDADO & Demandante \\
\hline 04 & $1083-2015$ LIMA & INFUNDADO & Demandante \\
\hline
\end{tabular}

Tabla 3

Otras causas que prevalecen

\begin{tabular}{|l|l|l|l|}
\hline $\mathrm{N}^{\circ}$ & $\mathrm{N}^{\circ}$ Expediente & Fallo & Favorecido \\
\hline 01 & $3581-2015$ LIMA & INFUNDADO & Demandado \\
\hline 02 & $2106-2015$ ICA & INFUNDADO & Demandado \\
\hline 03 & $484-2016$ LIMA & FUNDADO & Demandante \\
\hline
\end{tabular}

Se observa técnicamente el porcentaje de favorecimiento del demandante en el proceso de tercería de propiedad en sede casatoria, conforme se analiza de las sentencias casatorias correspondientes al periodo 2015 - 2016. 12, procesos sobre tercería, el $40 \%$ han favorecido a los demandantes, mientras que el $60 \%$ de los procesos han favorecido a los demandados.

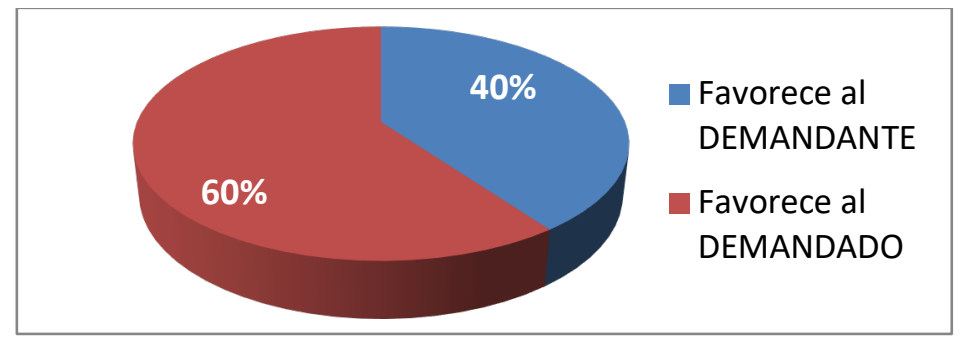

Figura 1

Favorecimiento del demandante en sus pretensiones conforme a sentencias 


\section{DISCUSIÓN}

Los principales fundamentos de las sentencias casatorias del periodo 2015-2016 sobre tercería de propiedad están referidos a la inscripción registral, la prevalencia del derecho real de la propiedad sobre el derecho personal y requisitos de procedibilidad del recurso de casación, implicando que los demandantes regularmente se hayan favorecido en sus pretensiones. La inscripción registral prevalece como problema de fondo en las pretensiones que se presentan en las tercerías de propiedad, tanto de los demandantes como de los demandados. Precisamente, al no ser obligatoria la inscripción registral para la transferencia de propiedad, se produce una serie de conflictos de interpretación por parte de los demandantes, demandados e incluso de los jueces. Si la inscripción registral fuese obligatoria en estos casos, consideramos que disminuirían considerablemente estos conflictos a nivel judicial. La prevalencia del derecho real (propiedad) sobre procesos de tercería de propiedad con pronunciamiento de fondo, tienen como principal fundamento la prevalencia del derecho real (propiedad) sobre el personal (crédito). Del análisis documental representados por los autos calificatorios y sentencias casatorias, si bien, el porcentaje mayoritario de favorecimiento les corresponde a los demandados, ambos se encuentran dentro del nivel regular. Por lo tanto, podemos afirmar que los demandantes en los procesos de tercería de propiedad en sede casatoria regularmente se han favorecido en sus pretensiones. Los principales fundamentos de las sentencias casatorias sobre tercería de propiedad están referidos a la inscripción registral, la prevalencia del derecho real de la propiedad sobre el derecho personal y requisitos de procedimiento del recurso de casación. Considerando que los procesos judiciales referidos a derechos de propiedad y medidas cautelares inscritas, muchas veces originan las demandas de tercerías de propiedad en el ámbito judicial; y en sede casatoria los demandantes muy poco logran los resultados favorables a sus intereses por no acreditar fehacientemente sus derechos con documento de fecha cierta, se recomienda a nivel judicial, se realicen capacitaciones permanentes a los magistrados sobre las diversas jurisprudencias relativas a la prevalencia del derecho real (propiedad) sobre el derecho personal (crédito), especialmente del VII Pleno Casatorio Civil publicado el 7 de diciembre de 2015 en el Diario Oficial El Peruano para que en la epata postulatoria del proceso de tercería se realice un "filtro" respecto a los documentos de fecha cierta que se ofrecen en la demanda y así evitar los largos procesos que coadyuvan a incrementar innecesariamente la carga procesal. Asimismo, que el Poder Judicial realice las coordinaciones necesarias con el Colegio de Notarios del Perú y demás entidades públicas pertinentes, para que cuando un Juez solicite se informe sobre la autenticidad o falsedad de un documento emitido, a la brevedad posible informe al Poder Judicial sobre dicha autenticidad o falsedad y no esperar interminables meses para que el Notario o funcionario informe sobre lo peticionado, originando que el proceso se dilate innecesariamente en perjuicio de los justiciables. Teniendo en cuenta las diversas jurisprudencias que determinan la prevalencia del derecho real (propiedad) sobre el derecho personal (crédito), y especialmente el VII Pleno Casatorio Civil sobre tercería de propiedad, se recomienda la modificación del artículo 535 del Código procesal Civil respecto a la admisibilidad y otros de la demanda de tercería. Con la modificación legal se buscará que los jueces en la etapa postulatoria le ponga interés 
preferente a los documentos de fecha cierta que ofrece el demandante tercerista de los cuales el juez podrá determinar si el documento es auténtico o no, en este último caso, podrá poner en conocimiento al Ministerio Público para que proceda conforme a sus atribuciones.

\section{REFERENCIAS BIBLIOGRÁFICAS}

Aguila Grados, G. (2010) Lecciones de Derecho Procesal Civil. Lima. Fondo Editorial de la Escuela de Altos Estudios Jurídicos EGACAL.

Arias-SchereiberPezet, M. (1998) Exégesis del Código Civil Peruano de 1984, Tomo II y IV. Lima: Gaceta Jurídica.

Atilio Cornejo, A. (1994) Derecho Registral. Bs. As. - Argentina: Edit. Astrea.

Balcázar Quiroz, J. (2016) Apuntes sobre los requisitos de admisibilidad de la casación civil alemana. Vox lure, Revista de la Facultad de Derecho y Ciencias Políticas de la Universidad Nacional Pedro Ruiz Gallo de Lambayeque Vol. 1 p. 51 - 63.

Bernales Ballesteros, E. (1999). La Constitución de 1993. Análisis Comparado. Lima: Editora RAO.

Cabanellas, G. (1981) Diccionario enciclopédico de Derecho usual. Bs. As. - Argentina: Editorial Heliasta,

Castillo Freyre, M. (1995) El Bien Materia del Contrato de Compra Venta Vol XIII. Lima: Fondo Editorial de la Pontificia Universidad Católica del Perú.

Celis Zapata, C. (2013) Casación Civil en el Perú. Universidad Inca Garcilaso de la Vega. Lima. Fondo Editorial de la UIGV.

Chirinos Soto, E. \& Chirinos Soto, F. (1997). Constitución de 1993. Lima. 4ta. Edición.

Diez-Picazo, L. (1996) Fundamentos del Derecho Civil Patrimonial. Madrid - España: Editorial Civitas.

Diez-Picazo, L. \& Gullón, A. Derecho de Cosas y Derecho Inmobiliario Registral. Madrid España: Edit. Tecnos S.A.

Duque Sánchez, J. (1978) Antecedentes históricos del recurso de Casación. Boletín de la Academia de Ciencias Políticas y Sociales / Academia de Ciencias Políticas y Sociales. Caracas. Vol. 37, No. 74

FranciskovicIngunza, B. \& Torres Angulo, C. (2017) La Corte Suprema ¿Tercera Instancia? García García, L. (2005) Comentario del Art. 2011 del C.C. Código Civil Comentado por los 100 mejores especialistas. Tomo X. Lima: Gaceta Jurídica Editores.

Gatti, E. \& Alterini, J. El Derecho Real. Bs. As. - Argentina: Edit. Abeledo Perrot.

Glave Mavila, C. (2012) El Recurso de Casación en el Perú. Revista Derecho \& Sociedad $N^{\circ}$ 38. Lima. Pontificia Universidad Católica del Perú.

Hernández, R. Fernández, C. \& Baptista, P. (2010). Metodologia de la Investigación Científica. México: Mc. Graw. Hill

Herrera Paulsen, D. (1984) Derecho Romano. Lima: 2da. Edición.

HinostrozaMinguez. A. (2000) El Embargo y Otras Medidas Cautelares. Lima. Editorial San Marcos.

La Puente Lavalle, M. Estudios sobre El Contrato de Compraventa. Lima: Gaceta Jurídica. Mariani de Vidal, M. (2004) Derechos Reales. Tomo I. Bs. As. - Argentina: Edit. Zavalia. 
Montenegro Cannon, M. (2000) La Cautela en el Proceso Civil Peruano. Lima: Gráfica Horizonte S.A.

Musto, N. (2000) Derechos Reales. Tomo I. Bs. As. - Argentina: Editorial Astrea.

Latorre Florido, C. (2004) El Recurso de Casación Civil: Antecedentes Históricos y Perfil Actual. Revista de Derecho $\mathrm{N}^{\circ} 12$. Santiago de Chile. Consejo de Defensa del Estado.

Ledesma Narváez, M. (2008) Comentarios al Código Procesal Civil. Tomo II. Lima: Gaceta Jurídica.

Palacio Pimentel, H. (1984) Manual de Derecho Civil. Tomo I. Lima: Editorial Huallaga.

Pasco Arauco, A. (2014) La tercería de propiedad frente a la hipoteca y el embargo. Cuando la dogmática puede más que el sentido común, la buena fe desaparece. Universidad Nacional Mayor de San Marcos. Extraído de:

Peñailillo Arévalo, D. (2006) Los Bienes, la Propiedad y otros Derechos Reales. Santiago de Chile: Editorial Jurídica de Chile.

Rioja Bermúdez, A. (2011) En defensa de la propiedad indebidamente embargada. Revista Oficial del Poder Judicial: Año 4 - 5, $N^{\circ} 6$ y N. ${ }^{\circ} 7$ / 2010-2011. Lima.

Rioja Bermúdez, A. (2011) La Casación en el Código Procesal Civil: Proyecto

modificatorio. Recuperado de:

Rivarola, E. (1984) Autor del desarrollo temático del término "Casación Civil" en la Enciclopedia Jurídica OMEBA. Bs. As. - Argentina. Editorial Driskill.

Sevilla Agurto, P. (2016) La regulación del recurso de casación en el Perú. Revista Gaceta Civil \& Procesal Civil N 34. Lima. Gaceta Jurídica. Extraído de:

Torres Vásquez, A. (2000) Código Civil. Comentarios y Jurisprudencia, concordancias, antecedentes, sumillas, legislación complementaria e índice analítico. Bogotá: Editorial Temis S.A.

Vásquez Rios, A. (2005) Derechos Reales. Tomo II. Lima: Editorial San Marcos.

Webb, R., Beurmann, D. \& Revilla, C. (2006) La Construcción del Derecho de Propiedad. Lima: Edit. Tarea Asociación Gráfica Educativa. 